\title{
Uncooled IR focal plane arrays: worldwide review and state-of-the-art at ULIS
}

\author{
by J.L. Tissot
}

ULIS, BP 21 - 38113 Veurey-Voroize, France - e-mail : jl.tissot@ulis-ir.com

\begin{abstract}
Uncooled infrared focal plane arrays are being developed for a wide range of thermal imaging applications. Developments are focused on improving the sensitivity to enable the possibility to manufacture high performance small pixel pitch detectors or radiometric detectors with internal "cold" shield. Commercial infrared is now a well-established business with several competitors. We review the state-of-art of the main competitors and we describe the work which is being done in France.

After the development of an amorphous silicon based uncooled microbolometer technology, LETI and ULIS, a subsidiary of SOFRADIR and CEA, are now working to make easier IRFPA integration into equipment in order to address a very large market. Achievement of this goal needs the integration of advanced functions on the focal plane and the decrease of manufacturing cost of IRFPA by decreasing the pixel pitch and simplifying the vacuum package. We present the new design for readout circuits taking into account the user needs by introducing analog to digital converter on the chip to provide 12 bits video digital output. We present also the introduction and the characterization of radiometric device obtained from $320 \times 240$ uncooled microbolometer arrays with a f/1.4 limited field of view. Then, we present the new ceramics microbolometer package developed to decrease the package cost of versatile smaller devices like the $160 \times 120$ pixels with a pitch of $35 \mu \mathrm{m}$.
\end{abstract}

\section{Introduction}

Uncooled microbolometer is going to take a large part of infrared imaging application business. Many developments are under progress and lightweight low power camera are already available making the uncooled microbolometer systems a viable alternative to many cooled IR systems.

Development efforts in uncooled focal plane array are now basically going in two directions:

- Arrays for military and high end commercial applications with the highest possible performance,

- Arrays for commercial applications with the lowest possible cost.

The goal of these efforts could be summarized in being able to decrease the pixel pitch keeping the same, or higher, level of performance. We present hereafter a brief worldwide overview of uncooled infrared focal plane array (IRFPA) developments.

\section{Uncooled infrared focal plane worldwide development}

Many worldwide developments are under progress mostly in USA and Europe and mainly supported by Departments of Defense concerned by the possibility to have low cost, low power infrared imaging camera for goggles, portable weapon sights or driver's vision enhancers. However, uncooled IR detector are typically "dual-use" since the same infrared modules could be used for both commercial and military applications.

\subsection{United States of America}

United States of America has been active in uncooled infrared focal plane arrays since the early 1980s with the work of Honeywell on vanadium oxide based microbolometers 
and Texas Instrument on ferroelectrics ceramics and amorphous silicon based detectors. Figure 1 shows important industrial organization evolution along the past twenty years.

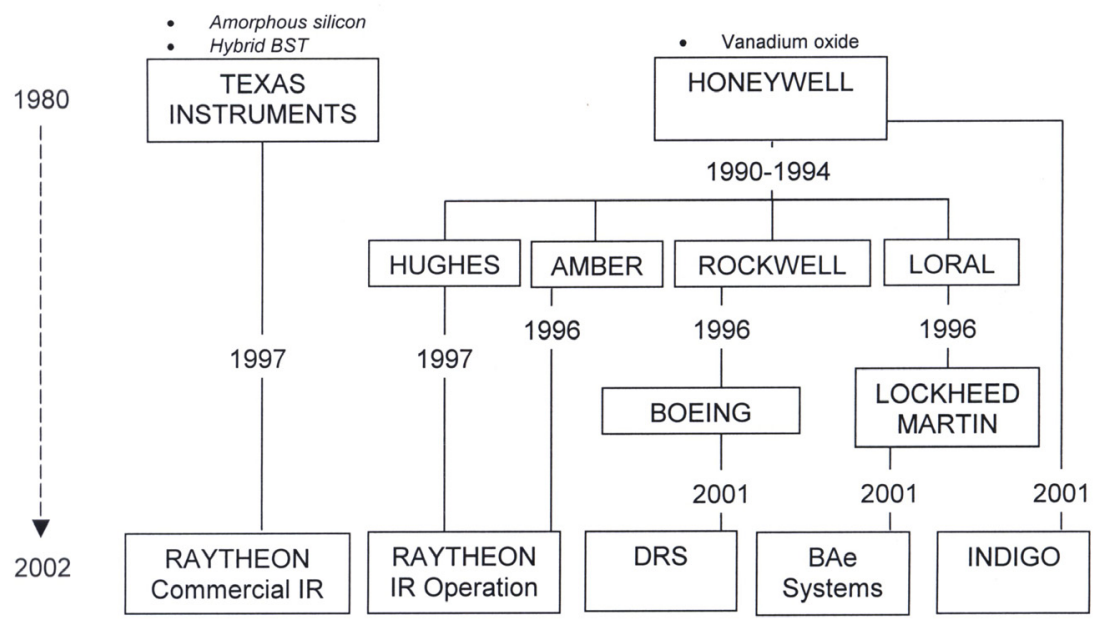

Fig. 1. Evolution of the main uncooled IRFPA industrial activities in USA from 1980 to 2002

Three technologies exist in USA:

Vanadium oxide technology previously developed by Honeywell and now by Raytheon Infrared Operation, DRS and BAe. High performance is already demonstrated with this technology and the challenge is now to be able to reduce the pixel pitch down to $25 \mu \mathrm{m}$. First results are shown by Raytheon [1] and DRS [2] with a pixel pitch of $25 \mu \mathrm{m}$ and a double deck structure and by BAe with a pixel pitch of $28 \mu \mathrm{m}$ and a more conventional simple deck structure [3]. The second challenge is to suppress the focal plane temperature stabilization in order to simplify the detector integration in camera. DRS has developed a radiometric version of its $320 \times 240$ pixels array and delivers its to infrared thermometry equipment manufacturer.

Ferroelectric BST technology previously developed by Texas Instrument and now by Raytheon Commercial Infrared. The hybrid detector structure of this technology limits the achievable thermal insulation of the absorber and thermometer and then the performance. However, Raytheon introduced a radiometric uncooled ferroelectric camera which makes this technology available to a broader range of users. Effort is now focused on the development of a monolithic structure in order to achieve higher thermal insulation. The difficulty is to realize very thin layer of ceramic material with high figure of merit [4].

Amorphous silicon technology previously developed by Texas Instruments [5] and now by Raytheon Commercial Infrared. This technology is much more oriented on low price commercial applications. An other company, ICC, is recently involved in another amorphous silicon microbolometer technology coming with a exclusive license agreement from DSTO (Australian Ministry of Defense).

\subsection{Europe}

In Europe, UK started the first uncooled development based on pyroelectrics detector and provided PZT-based focal plane arrays mostly for military applications. However these focal planes with $100 \times 100$ pixels were used in firefighting helmet camera. The 
second generation developed by BAe with QinetiQ, is now based on PST ceramics and $256 \times 128$ arrays are in production [6] and used in military infrared camera.

In Belgium, IMEC, using a strong expertise in silicon technology developed a Si-Ge polycrystalline bolometer technology industrialized by XeniC's [7] which is manufacturing linear arrays.

Activities of French CEA/LETI and ULIS teams are described below.

\subsection{Rest of the world}

Important developments are under progress in Canada and Japan. INO in Canada is very active in developing a vanadium oxide technology similar to those developed in USA [8]. In Japan, Mitsubishi develops with success an attractive silicon $\mathrm{p} / \mathrm{n}$ junction based technology. Beside, two years ago, NEC was working on vanadium oxide but no recent result are available. In Korea, Daewoo Electronics have made 256 × 256 double deck focal plane arrays [9] based on titanium microbolometer.

\section{Microbolometer technology development at CEA/LETI and ULIS}

In France, the development of the microbolometer technology is done by a unique structure composed of CEA/LETI for advanced microbolometer process development and ULIS for packaging development, process industrialization and IRFPA production.

CEA / LETI has been involved in Amorphous Silicon uncooled microbolometer development since 1992 with French MoD funding support. LETI chose from the very beginning to develop an amorphous silicon based monolithic microbolometer technology fully compatible with commercially available CMOS technology. "Monolithic architecture" and "silicon compatibility" are two key characteristics for being able to produce low cost IR detectors.

The first generation of amorphous silicon based microbolometer technology developed by CEA / LETI, is well mastered and very fast industrial transfer has been performed in 2000. Since this industrial transfer, CEA / LETI and ULIS are still working for improving the electro-optical performances and reducing the cost of the uncooled focal plane arrays. These developments are presented in the following paragraphs which cover the microbolometer and packaging technologies and the products.

\subsection{First generation technology under production}

Devices using the first generation technology are now under mass production at ULIS. Typical array characteristics and performances achieved with this technology are summarized in the following table for a $320 \times 240$ array with a pixel pitch of $45 \mu \mathrm{m}$.

Table I: Main characteristics of UL 0101 1device

\begin{tabular}{|lc|lc|}
\hline \multicolumn{1}{|c|}{ Parameter } & Value & \multicolumn{1}{c|}{ Parameter } & Value \\
\hline Pixel nb. & $320 \times 240$ & Pixel pitch & $45 \mu \mathrm{m}$ \\
Spectral response & $7-14 \mu \mathrm{m}$ & Fill factor & $>80 \%$ \\
NETD & $\leq 100 \mathrm{mK}$ & Operability & $>99 \%$ \\
Response & $6 \mathrm{mV} / \mathrm{K}$ & $\begin{array}{l}\text { Uncorrected response } \\
\text { uniformity }\end{array}$ & $<5 \%$ \\
$\begin{array}{l}\text { Typical scene } \\
\text { temperature range }\end{array}$ & $-10^{\circ} \mathrm{C}$ to $+50^{\circ} \mathrm{C}$ & $\begin{array}{l}\text { Nominal output rate } \\
\text { (60 Hz frame rate) }\end{array}$ & $5.5 \mathrm{MHz}$ \\
\hline
\end{tabular}

\subsection{Microbolometer technologies under development at CEA/LETI}

CEA / LETI and ULIS are working to increase the detector performance by pushing the design rules to improve the pixel thermal insulation. The key characteristics of the technology is the very thin amorphous silicon based micro bridge structure previously 
described [10] which leads to a small thermal time constant close to $4 \mathrm{~ms}$ in $50 \mu \mathrm{m}$ pixel pitch. To take advantage of this behavior, CEA / LETI has defined a new technological stack, fully compatible with the industrialized technology in order to improve thermal insulation at pixel level and to decrease 1/f noise level. This design enables an improvement of thermal resistance by a factor of 3 on $35 \mu \mathrm{m}$ pixel pitch. $1 / \mathrm{f}$ noise is reduced by increasing amorphous silicon thickness. However, pixel structure is still a one level electrode sustained by legs for thermal insulation purpose using the standard surface-micromachined single-level membrane technology. As far as we have a great margin with thermal time constant, thermal capacitance evolution is still acceptable unless thermal time constant is kept compatible with video frame rate.

This second generation microbolometer technology is now under mastering in CEA/LETI [11] pilot line and will be transfer to ULIS during second part of this year. Then the production will ramp up next year using the new ULIS facilities. Improvement based on pixel design and readout integrated circuit design is still forecasted for the future.

\section{Product development at ULIS}

The product roadmap takes into account array formats needed by the applications with the smallest pixel pitch compatible with the required performances and the available microbolometer technology.

\section{$4.145 \mu \mathrm{m}$ pixel pitch devices}

\section{Standard product:}

Production of standard product UL 01011 is now ramping up to produce near thousand detector per month next year. Up to now SOFRADIR / ULIS has been delivering more than thousand detectors since the transfer of technology from CEA / LETI. NETD down to $70 \mathrm{mK}$ for $\mathrm{F} / 1$ aperture and $50 \mathrm{~Hz}$ frame rate, has been demonstrated for $295 \mathrm{~K}$ operating temperature. The main characteristic of our product is the low detector time constant enabling operation at $60 \mathrm{~Hz}$ frame rates without any video artifacts on fast motion objects in the scene.

\section{Radiometric product:}

A radiometric version has been derived from the standard product using the same device integrated with an internal shield limiting the field of view to f/1.4 (see Figure 2). The shield aperture is $16.3 \mathrm{~mm}$ in diameter and positioned at $22.77 \mathrm{~mm}$ above focal plane. These new devices exhibit uniform noise and responsivity characteristics. A histogram of the NETD data for the first array is presented in Figure 3. The mean NETD is $147 \mathrm{mK}$ and the same microbolometer bias is used for $20^{\circ} \mathrm{C}$ to $35^{\circ} \mathrm{C}$ or $20^{\circ} \mathrm{C}$ to $100^{\circ} \mathrm{C}$ blackbody reference temperatures. Fixed pattern noise measured for $65^{\circ} \mathrm{C}$ background after a two points correction at $20^{\circ} \mathrm{C}$ and $100^{\circ} \mathrm{C}(584 \mu \mathrm{V} \mathrm{rms})$ is close to temporal noise (509 $\mu \mathrm{V} \mathrm{rms),} \mathrm{(see} \mathrm{figure} \mathrm{4).} \mathrm{Extensive} \mathrm{characterization} \mathrm{procedure} \mathrm{is} \mathrm{under} \mathrm{progress} \mathrm{to}$ demonstrate its stability against environment temperature fluctuations.
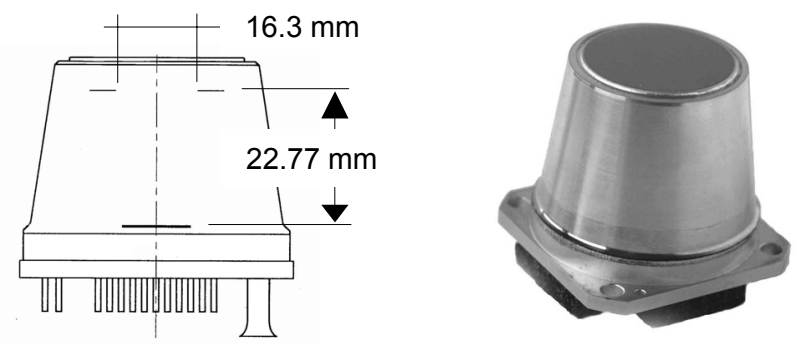

Fig. 2. Radiometric version of the $320 \times 240 / 45 \mu \mathrm{m}$ device 


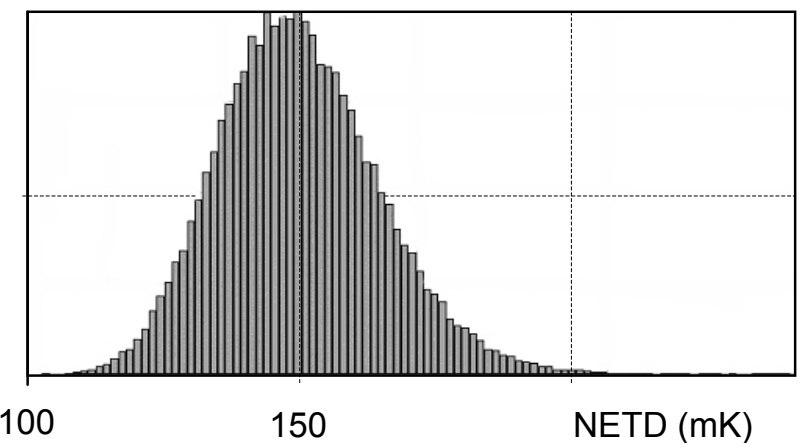

Fig. 3. NETD distribution (f/1.4, $30 \mathrm{~Hz}, 300 \mathrm{~K})$

\section{2 $35 \mu \mathrm{m}$ pixel pitch devices}

The development and the mastering of the second generation microbolometer technology enable to decrease the pixel pitch to $35 \mu \mathrm{m}$ keeping the same level of performance. Taking profit from this possibility, we have designed $160 \times 120$ and $388 \times$ $2842 \mathrm{D}$ arrays with a number of on-chip innovative features to simplify the use of these focal planes keeping a very small silicon ROIC area down to $0.7 \mathrm{~cm}^{2}$ for the $160 \times 120$ array, in order to reduce wafer-level processing costs per die. This new $160 \times 120$ is designed to fulfill medium resolution, low cost applications. One of the most promising function is the possibility to adjust the skimming of the common mode current for each pixel instead of each column like it is done in standard product. At power on, the detector acquires first the pixel non uniformity coefficients for each pixel and secondly stores them in on-chip memory and then performs the current compensation during the following images readout sequences. This automatic mode of operation could be changed to an external driving mode with non uniformity coefficients stored in an external memory (see figure 5). Beside, the video output will be available from analogical or digital format with on-chip 12 bits $(2 \times 6)$ ADC. Most of the bias are generated inside of the ROIC for friendly user operation.

This design is also used for a new $384 \times 288$ array which can be used as a $320 \times 240$ to comply with US standard.
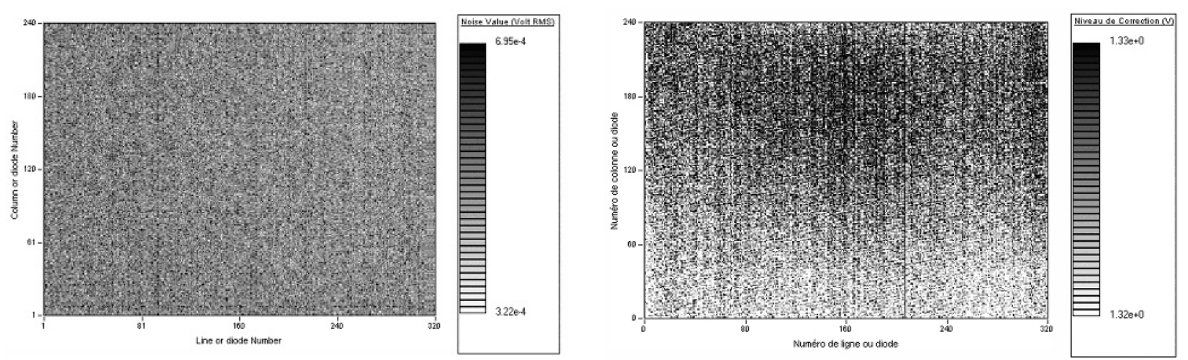

Fig. 4. Temporal noise map and Fixed pattern noise map 


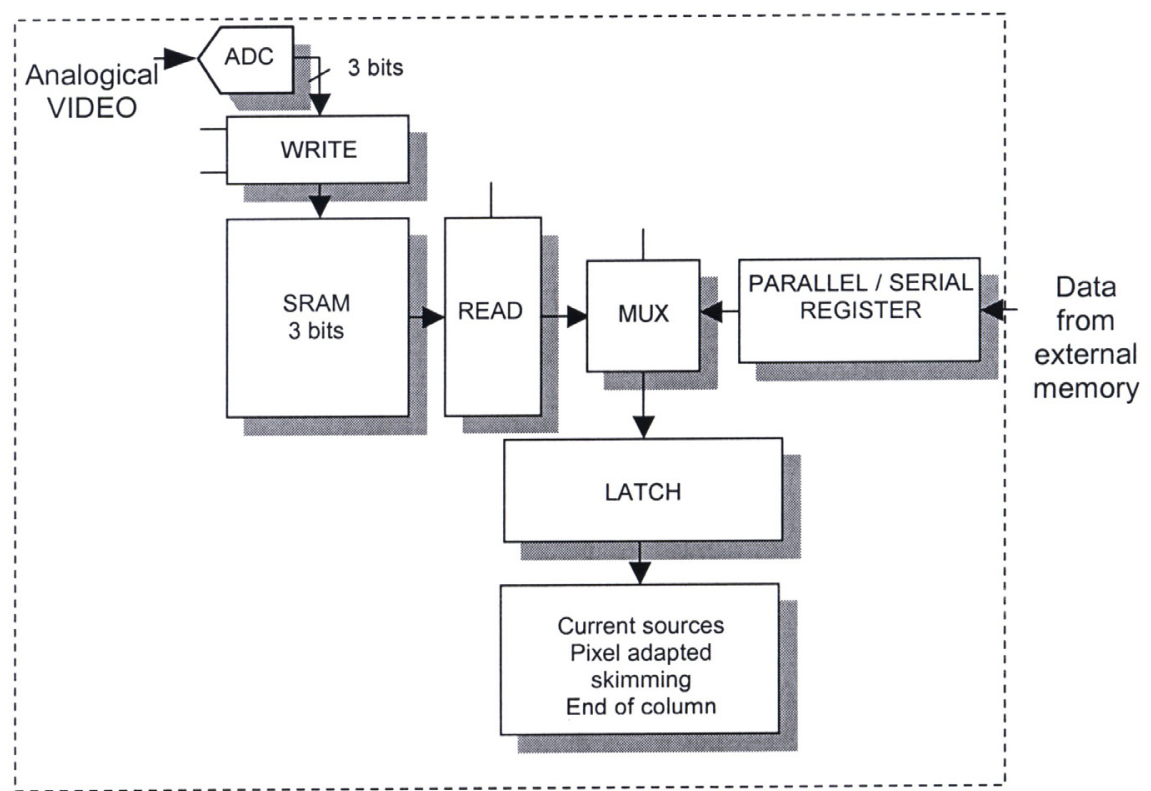

Fig. 5. Synoptic of $160 \times 120$ array skimming operation

\section{Packaging development}

The development of low cost silicon-based microbolometer chip has created a new demand for packaging technology to provide smaller, more cost effective solutions to house the devices. Microbolometer packaging is one of the most costly parts of the whole manufacturing process. Different options could be use for microbolometer packaging and each have relative cost and assembly issues which need to be considered for volume application.

\subsection{Metallic package}

Metallic package is the first generation of package used to integrate microbolometer chip. Their mechanical strength is well adapted to harsh environment and their design could be easily adapted to applications. But its cost remains a large part of the total detector cost and with the possibility to decrease detector chip size, this part will be greater in the future. In consequence a less expensive package technology is under development to integrate the new generation of detector chip.

\subsection{Ceramic package}

Microbolometer ceramic packages are designed using available technologies developed and produced for integrated circuit. Only the chip carrier and window carrier assembling processes are adapted to take into account the required over 10 year life time under vacuum. Automatic assembling machine will be used to decrease manufacturing cost by suppressing manual operations and increasing throughput.

\subsection{Wafer level packaging}

Beside, these advanced packaging techniques, CEA / LETI is working on the development of wafer level packaging in order to achieve the ultimate reduced 
manufacturing cost. This goal will be achieved by developing a silicon package (chip carrier and IR window [12] instead of welding infrared window on top of microbolometer array wafers as developed elsewhere (see Figure 6). This last technique involves an increase of the microbolometer chip area which is not compatible with the manufacturing cost goal we have.
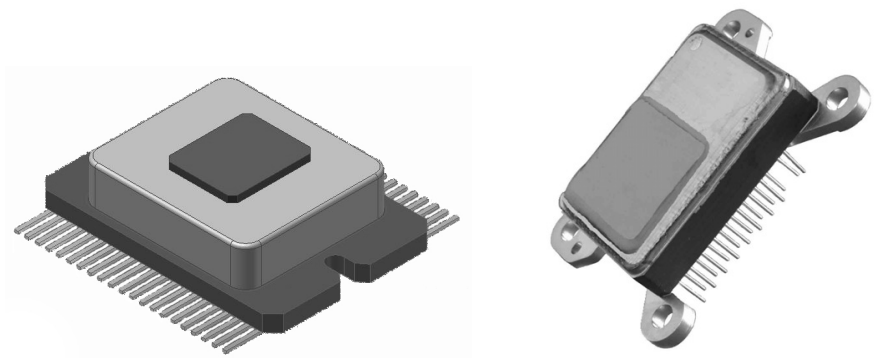

Fig. 6. Ceramic packages for $160 \times 120$ (left) and $320 \times 240$ arrays (right)

\section{Conclusions}

We obtained significant results in term of signal to noise ratio improvement. We took advantage of our low thermal time constant characteristic on standard technology that paves the way to increase thermal resistance keeping a low thermal time constant compatible with video frame rate up to $100 \mathrm{~Hz}$. A new pixel design participates to reduce the noise level enabling the possibility to achieve high performance in spite of pixel pitch reduction. In consequence, signal to noise ratio has shown a fivefold improvement compared to standard technology and NETD down to $30 \mathrm{mK}$ has been demonstrated on laboratory prototype with $35 \mu \mathrm{m}$ pixel pitch. Development of radiometric product shows high level on uniformity achieved with standard technology. New package technologies and advance $35 \mu \mathrm{m}$ pixel pitch detector are under mastering in order to decrease detector cost.

\section{Acknowledgments}

The authors would like to thank the DGA/DTCO and the Ministère de l'Economie, des Finances et de l'Industrie for supporting these studies and the Uncooled Teams of LETI LIR and ULIS who took part in them. We also thank the Sofradir staff for their contribution.

\section{REFERENCES}

[1] D. Murphy, M. Ray, R. Wyles, J. Asbrock, N. Lum, J. Wyles, C. Hewitt, A. Kennedy, D. Van Lue, J. Anderson, D. Bradley, R. Chin, T. Kostrzewa, "High Sensitivity $25 \mu \mathrm{m}$ Microbolometer FPAs", Proceeding of SPIE Vol. 4721, pp. 99-110, (2002), "infrared Detectors and Focal Plane Arrays VII", Orlando, FI, USA

[2] P.E. Howard, J.E. Clarke, A.C. Ionescu, C. Li, J.C. Stevens, "DRS U6000 640 x 480 Vox Uncooled IR Focal Plane", Proceeding of SPIE Vol. 4721, pp. 48-55, (2002), "infrared Detectors and Focal Plane Arrays VII", Orlando, FI, USA

[3] B.S. Backer, N. Butler, M. Kohin, M. Gurnee, J.T. Whitwam, T. Breen, "Recent improvements and developments in Uncooled systems at Bae SYSTEMS North America", Proceeding of SPIE Vol. 4721, pp. 83-90, (2002), "infrared Detectors and Focal Plane Arrays VII", Orlando, FI, USA

[4] C.M. Hanson, H.R. Beratan, "Thin-film ferroelectrics : breakthrough", Proceeding of SPIE Vol. 4721, pp. 91-98, (2002), "infrared Detectors and Focal Plane Arrays VII", Orlando, FI, USA

[5] United State Patent N ${ }^{\circ} 5,367,167$, Nov. 22, 1994 
[6] M.A. Todd, P.P. Donohue, R. Watton, D.J. Williams, C.J. Anthony, M.G. Blamire, "High performance ferroelectric and magnetoresistive materials for next-generation thermal detector arrays", Infrared Detectors and Focal Plane Arrays VII, to be published in SPIE Vol. 4721, 2002

[7] V.N. Leonov, N.A. Perova, J.P. Vermeiren, B. Grietens, C. Goessens, P. De Moor, C.A. Van Hoof, "Optimization of design and technology for uncooled poly-SiGe icrobolometer arrays", Proceeding of SPIE Vol. 4721, pp. 122-133, (2002), "infrared Detectors and Focal Plane Arrays VII", Orlando, FI, USA

[8] T.D. Pope, H. Jerominek, C. Alain, C. Cayer, B. Tremblay, C. Grenier, P.A. Topart, S. LeClair, F. Picard, C. Larouche, B. Boulager, A. Martel, Y. Desroches, "Commercial and custom $160 \times 120,251 \times 1$ and 512 × 3 pixel bolometric FPAs", Proceeding of SPIE Vol. 4721, pp. 64-74, (2002), "infrared Detectors and Focal Plane Arrays VII", Orlando, FI, USA

[9] Hyung-Kew Lee et al, "A High Fill-Factor IR Bolometer Using Multi-Level Electrothermal Structures", IEEE Trans. Electron Devices (USA), vol. 46, n7, p.1489-91 (July 1999)

[10] C. Vedel, J.L. Martin, J.L. Ouvrier-Buffet, J.L. Tissot, M. Vilain, J.J. Yon, "Amorphous silicon based uncooled microbolometer IRFPA", SPIE Vol. 3698, "Infrared Technology and Application XXV", Orlando, FI. USA, 1999

[11] E. Mottin, A. Bain, J.L. Martin, J.L. Ouvrier-Buffet, J.J. Yon, J.P. Chatard, J.L. Tissot, "Uncooled amorphous silicon technology : high performance achievement and future trends", Infrared Detectors and Focal Plane Arrays VII, to be published in SPIE Vol. 4721,2002

[12] E. Mottin, J.L. Martin, J.L. Ouvrier-Buffet, M. Vilain, A. Bain, J.J. Yon, J.L. Tissot, J.P. Chatard, "Enhanced amorphous silicon technology for 320 x 240 microbolometer arrays with a pitch of $35 \mu \mathrm{m}$ ",Infrared Detectors and Focal Plane Arrays VI, SPIE Vol. 4369, 2001 